\title{
ORIGINAL ARTICLE \\ No evidence for MHC class II-based non-random mating at the gametic haplotype in Atlantic salmon
}

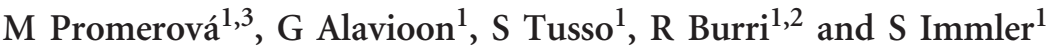

Genes of the major histocompatibility complex $(\mathrm{MHC})$ are a likely target of mate choice because of their role in inbreeding avoidance and potential benefits for offspring immunocompetence. Evidence for female choice for complementary $\mathrm{MHC}$ alleles among competing males exists both for the pre- and the postmating stages. However, it remains unclear whether the latter may involve non-random fusion of gametes depending on gametic haplotypes resulting in transmission ratio distortion or non-random sequence divergence among fused gametes. We tested whether non-random gametic fusion of MHC-II haplotypes occurs in Atlantic salmon Salmo salar. We performed in vitro fertilizations that excluded interindividual sperm competition using a split family design with large clutch sample sizes to test for a possible role of the gametic haplotype in mate choice. We sequenced two MHC-II loci in 50 embryos per clutch to assess allelic frequencies and sequence divergence. We found no evidence for transmission ratio distortion at two linked $\mathrm{MHC}$-II loci, nor for non-random gamete fusion with respect to $\mathrm{MHC}$-II alleles. Our findings suggest that the gametic MHC-II haplotypes play no role in gamete association in Atlantic salmon and that earlier findings of MHC-based mate choice most likely reflect choice among diploid genotypes. We discuss possible explanations for these findings and how they differ from findings in mammals.
\end{abstract}

Heredity (2017) 118, 563-567; doi:10.1038/hdy.2016.129; published online 18 January 2017

\section{INTRODUCTION}

Major histocompatibility complex (MHC) molecules play a crucial role in activating the adaptive immune system in jawed vertebrates (Klein, 1986) and studies across taxa commonly report a direct link between MHC and fitness (reviewed in Sommer, 2005; Piertney and Oliver, 2006). Moreover, MHC genes have been shown to be involved in mate choice in different species (reviewed in Milinski, 2014). MHC-based mate choice has been suggested to provide fitness benefits in two ways. First, females could benefit from mating with males bearing resistance alleles to common or severe pathogens in a given population ('good genes' hypothesis), ensuring enhanced resistance to those pathogens in their progeny (Ekblom et al., 2004; Richardson et al., 2005; Eizaguirre et al., 2009; Cutrera et al., 2012). Second, as individual MHC molecules bind only a specific subset of pathogens, it should be advantageous to maximize heterozygosity in offspring (Hughes and Nei, 1988; Lenz et al., 2009). In this case, females would choose mates non-randomly to complement their own genotypes ('complementary genes hypothesis'; see, for example, Wedekind et al., 1995; Penn and Potts, 1998; Consuegra and Garcia de Leaniz, 2008).

Despite a widespread role of MHC in mate choice, the mechanisms of recognizing a potential mating partner based on MHC genotype are not well understood. In mammals, birds and fish, MHC molecules are believed to influence body odor and hence odor preferences during mate choice (see, for example, Yamazaki et al., 1988; Potts et al., 1991; Wedekind et al., 1995; Carroll et al., 2002; Richardson et al., 2005). Studies in Atlantic salmon, Salmo salar, confirm that MHC-II diversity is linked to disease resistance (Lohm et al., 2002) and that both
MHC-I and -II affect mate choice (Landry et al., 2001; Consuegra and Garcia de Leaniz, 2008; Yeates et al., 2009). Generally, MHC-I diversity is involved in $\mathrm{T}$ cell-mediated response and resistance to viruses, whereas MHC-II diversity affects resistance to bacteria. Interestingly, the direction of mate choice appears to differ between the two MHC classes. A study using in vitro fertilization (IVF) to induce competition between sperm of two males showed that eggs were preferentially fertilized by males with genetically similar MHC-I genotype (Yeates et al., 2009). In contrast, MHC-II showed significantly higher genetic diversity than expected under random mating in the offspring of wild salmon, suggesting premating mate choice for heterozygosity at the individual level (Landry et al., 2001). However, the inconsistencies across studies could also hint at the fact that MHC alleles may play an important role at the postmating prezygotic stages.

In externally fertilizing organisms such as fish, an MHC recognition mechanism at the postmating level is thought to be particularly important as females have reduced control over fertilization because of group spawnings or males assuming alternative mating strategies (Taborsky, 1998). In addition, external fertilization impedes the potential maternal impact on the offspring MHC genotype via abortion as it is found for example in mammals (Wedekind, 1994). The question thus arises of how eggs recognize MHC allele composition in sperm. Studies on sperm competition between sperm from different males suggest the occurrence of MHC-dependent mate choice even after mating (cryptic female choice; Schwensow et al., 2008; Yeates et al., 2009; Lovlie et al., 2013). Although these studies tested for a role of MHC in mate choice at the postmating level, they

\footnotetext{
${ }^{1}$ Department of Evolutionary Biology, EBC, Uppsala University, Uppsala, Sweden and ${ }^{2}$ Department of Population Ecology, Friedrich Schiller University Jena, Jena, Germany Correspondence: Dr M Promerová or Dr S Immler, Department of Evolutionary Biology, Uppsala University, Norbyvägen $18 D$, SE-752 36 Uppsala, Sweden. E-mail: promerova@seznam.cz or simone.immler@ebc.uu.se

${ }^{3}$ Current address: Department of Archaeogenetics, Max Planck Institute for the Science of Human History, Jena, Germany. Received 10 August 2016; revised 17 November 2016; accepted 5 December 2016; published online 18 January 2017
} 
reveal no information about a possible mechanism at the level of the gametic haplotype. There is no clear evidence for the (haploid) expression of the MHC repertoire on the sperm cell surface. Although some studies on human spermatozoa show haploid expression of MHC (see, for example, Halim and Festenstein, 1975; Arnaizvillena and Festenstein, 1976), others find no MHC antigens (Haas and Nahhas, 1986) or report inconsistent results (Kurpisz et al., 1987). A second, less explored possibility for selective fertilization, which so far has only been tested in mammals, arises after membrane penetration when the egg could choose between its two remaining haplotypes to optimize complementarity with that of the penetrating sperm genotype (Agulnik et al., 1993; Wedekind et al. 1996).

To study the potential role of non-random gamete fusion in postmating mate choice, we investigated transmission ratio distortion and non-random haplotype divergence within gametes at two tightly linked functional MHC-II loci (DAA and DAB) in Atlantic salmon. The two loci represent the only copies of MHC-IIA and MHC-IIB in salmon, and their variation has been extensively described in the past (Langefors et al., 2000; Stet et al., 2002; Gomez et al., 2010). We performed IVF assays with sperm from single males thus limiting potential assortative fusion among gametes exclusively to the haploid stage. Our aim was to answer three questions: (1) Are any MHC-II alleles transmitted to offspring in a non-Mendelian manner? (2) Is there any distortion in genotype frequencies? (3) Do we observe different sequence divergence in the offspring than expected under random gamete fusion?

\section{MATERIALS AND METHODS}

\section{Sampling and IVF}

Fifteen males (length: $77.6 \mathrm{~cm} \pm 11.3$ s.d., weight: $4.52 \mathrm{~kg} \pm 1.94$ s.d.) and 15 females (length: $92.2 \mathrm{~cm} \pm 9.5$ s.d., weight: $8.67 \mathrm{~kg} \pm 2.59$ s.d.) were randomly sampled during commercial stripping of wild S. salar at the Fishery Research Station in Älvkarleby, Sweden. Fish were anaesthetized with a solution of Tricaine Methanesulfonate (MS 222, Sigma-Aldrich, Stockholm, Sweden) during gamete collection. IVF was performed under laboratory conditions. Ejaculates and eggs were collected into plastic containers by carefully avoiding premature contact with water and kept cool on ice until IVFs (not more than $2 \mathrm{~h}$ after collection). For fertilization, on average $291 \pm 56$ eggs per subclutch were placed in a dry glass beaker. Eggs remained in ovarian fluid for IVFs. We then added $100 \mu \mathrm{l}$ of ejaculate and $200 \mathrm{ml}$ of river water $\left(\sim 3^{\circ} \mathrm{C}\right)$ for activation of the gametes. Each individual was mated three times independently, that is, eggs were fertilized by three different males and vice versa, resulting in a total of 45 clutches in five full factorial $3 \times 3$ blocks, where males and females were randomly assigned to each block. Fertilized eggs were allowed to harden for $5 \mathrm{~min}$, excessive sperm were washed away and eggs were disinfected with poly vinyl pyrrolidone iodine solution (Frantsi and Withey, 1972) and incubated in the hatchery system for 4 months at river water temperature (average water temperature $3.2^{\circ} \mathrm{C} \pm 0.2$ s.d.) until the eyes of the embryos became visible because of black pigmentation ('eyed eggs'). The opaque colour of salmon eggs and the necessity for eggs to lay still during early development prevents the assessment of fertilization on live eggs before the eyed stage. Eggs turning white were removed throughout incubation to avoid fungal growth ( 7 eggs \pm 10 s.d. per clutch; except for three clutches from one male with low fertilization success). Embryos were killed and stored in $96 \%$ ethanol at $+4{ }^{\circ} \mathrm{C}$ until DNA extraction.

\section{Genetic analysis}

Genomic DNA was extracted from the ejaculate of the 15 males used for IVFs following a protocol specifically designed for DNA extraction from sperm (Qiagen: Purification of total DNA from animal sperm using the DNeasy Blood \& Tissue Kit; protocol 2) and from 50 embryos per clutch (2250 embryos in total) using the DNeasy Blood \& Tissue Kit (Qiagen, Sollentuna, Sweden) according to the manufacturer's instructions. Exon 2 of DAA (303 bp) was amplified using primers for S. salar DAA published by Gomez et al. (2010) and DAB
(311 bp) using the primers TVS4501 and AL1002 (Langefors et al., 2000). PCR products from all 15 males were cloned into bacterial vectors (pGEM T-easy, Promega, Stockholm, Sweden) and Sanger sequenced to estimate paternal genotypes.

\section{Embryo genotyping}

Because of the length of the DAA and DAB loci, we amplified and sequenced the two loci separately. Both forward and reverse primers were tagged using an $8 \mathrm{bp}$ barcode on the $5^{\prime}$-end for sample identification after pooling for sequencing. The PCR consisted of $0.2 \mu \mathrm{M}$ of each tagged forward and reverse primer, $0.2 \mathrm{~mm}$ dNTPs, $2 \mathrm{~mm} \mathrm{MgCl}_{2}, 1 \times$ Gold PCR buffer, 1 unit of AmpliTaq Gold polymerase (Life Technologies, Stockholm, Sweden), $1 \mu \mathrm{l}$ DNA and $\mathrm{dd}_{2} \mathrm{O}$ to a final volume of $25 \mu \mathrm{l}$. Cycling conditions were: $94{ }^{\circ} \mathrm{C}$ for $5 \mathrm{~min}$, followed by 30 cycles of $94^{\circ} \mathrm{C}$ for $40 \mathrm{~s}, 58^{\circ} \mathrm{C}$ for $30 \mathrm{~s}$ and $72{ }^{\circ} \mathrm{C}$ for $1 \mathrm{~min}$, and a final extension step at $72^{\circ} \mathrm{C}$ for $7 \mathrm{~min}$. Amplification products were visualized in $1.5 \%$ agarose gels stained with GelRed (Biotium, Stockholm, Sweden). PCR products were then arbitrarily pooled by 16 samples (pre-pools) according to gel band intensity as follows: $5 \mu \mathrm{l}$ of strong products and $10 \mu \mathrm{l}$ of weak products, and purified using the QIAquick PCR purification kit (Qiagen) according to the manufacturer's instruction. DNA concentration of the purified pre-pools was individually measured using Nanodrop 2000 (Thermo Scientific, Stockholm, Sweden) and then equimolarly pooled into six final samples (three for DAA and three for DAB). These final pooled samples were processed as separate paired-end libraries $(2 \times 300 \mathrm{bp})$ during the sequencing process using the MiSeq platform (Illumina, Eindhoven, The Netherlands) at the National Genomics Infrastructure at SciLifeLab, Uppsala, Sweden. DAA and DAB amplicons were sequenced on two different MiSeq lanes with families being randomized across the two lanes.

To estimate genotyping reliability for both DAA and DAB, 186 samples $(\sim 8 \%)$ were run in duplicate, with differently tagged primers and in different library preparations. In addition, 10 samples were repeated in 5 different PCRs each, with different primer tags, but with the same library preparation. The results of the replicates fully support the reliability and robustness of the genotyping method.

\section{Genotype extraction and sequence analysis}

Paired reads were merged using FLASH (Magoč and Salzberg, 2011) and sorted by individual samples based on tagged primer combinations. The individual haplotypes and genotypes were identified using python scripts based on the coverage and frequency of each candidate haplotype. An individual was considered homozygote if the frequency of the most abundant haplotype was $>0.9$, and heterozygote if the frequency of the two most abundant haplotypes were between 0.45 and 0.55 . The maternal genotypes were reconstructed using the information from the offspring and paternal genotypes. The identity of the haplotype sequences was determined by BLAST with referent sequences from GenBank. Relevant scripts can be downloaded from https://github.com/ situssog/Promerova_et_al_2016.

Families with two homozygous parents (three cases) or with both parents heterozygous for the same alleles (one case) were excluded from the analyses, as it was not possible to distinguish between paternal or maternal origin of the alleles. The putative peptide-binding region sites were established based on Brown et al. (1993) and Dixon et al. (1996). Genetic distances between parental haplotypes were calculated as pairwise nucleotide sequence divergence using the Jukes-Cantor model, with uniform rates among sites and pairwise deletion, and pairwise amino-acid sequence divergence using Poisson distribution in Mega v.5 (Tamura et al., 2011).

\section{Statistical analysis}

Exact binomial tests were used to assess whether any of the alleles show transmission ratio distortion. For each allele, information from all families was combined to maximize statistical power. We compared the number of transmitted (number of offspring that inherited the particular allele) and non-transmitted (number of offspring not inheriting this allele) alleles across all families in which at least one of the parents was heterozygous. The expected proportion of transmitted to non-transmitted alleles was 0.5 (the predicted value under Mendelian inheritance; see Supplementary Table S1 for family 
values). To correct for multiple testing we used false discovery rate correction. The analysis was carried out separately for alleles of paternal and maternal origin.

According to the null hypothesis of no distortion, we expect four possible allelic combinations within a family with frequencies of 0.25 if both parents are heterozygous for a given locus. If only one of the parents is heterozygous, the two possible allelic combinations in the offspring should not differ from 0.5. Whether the observed frequencies follow expected values was tested with the $\chi^{2}$ test of goodness of fit and subsequent false discovery rate correction. Monte Carlo simulations with 10000 iterations were employed to estimate expected genotype frequencies across the 'population' in our data set.

In addition, we tested whether eggs were preferentially fertilized by sperm carrying MHC-II haplotypes different or similar to their own. We compared the offspring's expected mean MHC divergence and its variance under random fertilization by one of two available sperm haplotypes (obtained by Monte Carlo simulations with 10000 iterations) to the observed mean MHC divergence and the observed variance. The same test was performed for the nucleotide and amino-acid sequences of the whole exon 2 and then for only the peptide-binding region sites. All simulations and statistical tests were performed in $\mathrm{R}$ version 3.1.2 (R Core Team, 2014).

\section{RESULTS}

With 9 and 11 alleles, the genetic diversity detected at the DAA and DAB loci observed here was comparable to previous studies (Langefors et al., 2000; Stet et al., 2002; Gomez et al., 2010). The sequences of these alleles differed by 2 to 12 amino acids at DAA and 3 to 19 amino acids at DAB.

The most common allele at the DAA locus $\left(\mathrm{DAA}^{*} 0201\right)$ showed preferential transmission when inherited from females $(P=0.021$, $N=15$ families and 750 informative meioses, 407 transmitted and 343 non-transmitted; Table 1). However, this result was not significant after false discovery rate correction (threshold 0.006). For the same allele when inherited from males, there was a nonsignificant trend in the opposite direction $(P=0.12, N=18$ families and 896 informative meiosis, 424 transmitted and 472 non-transmitted). At the DAB locus, none of the allele frequencies showed significant distortion. None of the alleles of either locus showed transmission ratio distortion when tested for both parental sexes combined.

When testing for distortion in genotype frequencies within families, none of the 40 included Atlantic salmon families showed significant deviations from expected genotype frequencies after false discovery rate correction, even though in three families the uncorrected $P$-value was significant $(P=0.01-0.025$; Supplementary Table S2). We did not observe any deviation from expected genotype frequencies across the whole data set (Supplementary Table S3). Results were the same for both DAA and DAB.

Table 2 Mean sequence divergence in offspring measured as nucleotide and amino-acid distance and its variance for the whole exon 2 sequence and for PBR sites only

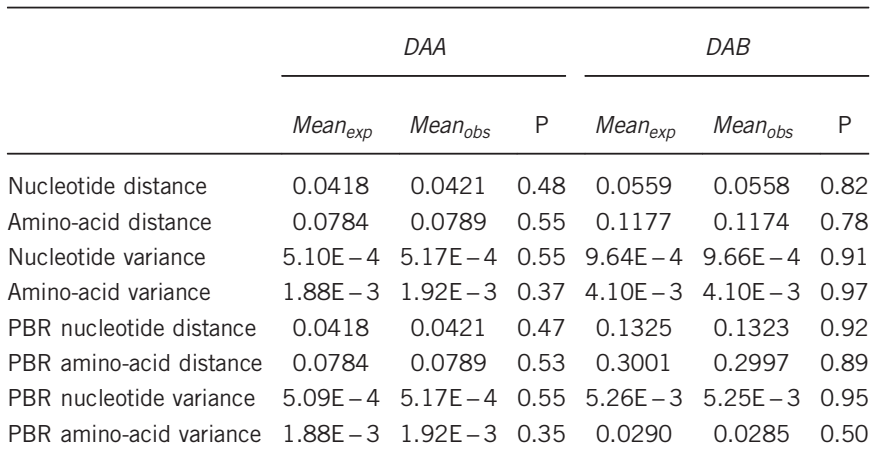

Abbreviations: Mean $_{\text {exp }}$, expected mean value; Mean $_{\text {obs, }}$ observed mean value; PBR, peptidebinding region.

Expected mean values were obtained by Monte Carlo simulations with 10000 iterations.

$P$ indicates $P$-values before correction for false discovery rate (Taborsky, 1998).

Table 1 Transmission of exon 2 DAA and DAB, MHC-II from males and from females

\begin{tabular}{|c|c|c|c|c|c|c|}
\hline Alleles & Transmitted from males & Non-transmitted from males & $\mathrm{P}_{M}$ & Transmitted from females & Non-transmitted from females & $\mathrm{P}_{F}$ \\
\hline$D A A * 0201$ & 424 & 472 & 0.12 & 407 & 343 & 0.02 \\
\hline $\mathrm{DAA}^{*} 0302$ & 195 & 205 & 0.65 & 201 & 197 & 0.88 \\
\hline$D A A^{* 0802}$ & 231 & 217 & 0.54 & 218 & 230 & 0.60 \\
\hline DAA*allele $01^{a}$ & 83 & 67 & 0.22 & 70 & 80 & 0.46 \\
\hline $\mathrm{DAA}^{*} 1001$ & 130 & 120 & 0.57 & 196 & 202 & 0.80 \\
\hline DAA*0303 & 159 & 141 & 0.33 & 69 & 79 & 0.46 \\
\hline $\mathrm{DAA}^{*}$ allele02a & 66 & 84 & 0.16 & 77 & 73 & 0.81 \\
\hline$D A A^{*} 0501$ & 158 & 140 & 0.32 & - & - & - \\
\hline$D A A^{*} 0901$ & - & - & - & 208 & 242 & 0.12 \\
\hline DAB-DB1 ${ }^{*} 06$ & 200 & 200 & 1.00 & 218 & 232 & 0.54 \\
\hline DAB-DB1*10 & 198 & 202 & 0.88 & 311 & 289 & 0.39 \\
\hline DAB-DB1 * 11 & 210 & 240 & 0.17 & 246 & 204 & 0.05 \\
\hline DAB-DB $1 * 17$ & 195 & 205 & 0.65 & 201 & 197 & 0.88 \\
\hline $\mathrm{DAB} * 0403$ & 159 & 141 & 0.33 & 71 & 79 & 0.57 \\
\hline DAB-DB $1 * 08$ & 130 & 120 & 0.57 & 50 & 48 & 0.92 \\
\hline DAB-DB $1 * 12$ & 84 & 66 & 0.16 & 70 & 80 & 0.46 \\
\hline DAB-DB $1 * 01$ & 66 & 84 & 0.16 & 73 & 77 & 0.81 \\
\hline DAB-DB1*03 & 158 & 142 & 0.39 & - & - & - \\
\hline DAB-DB1 *09 & - & - & - & 146 & 154 & 0.69 \\
\hline DAB-DB1 * 13 & - & - & - & 208 & 242 & 0.12 \\
\hline
\end{tabular}

Abbreviations: MHC-II, major histocompatibility complex class II; $P_{\mathrm{F}}$, uncorrected $P$-values for females; $P_{\mathrm{M}}$, uncorrected $P$-values for males.

Numbers indicate the total number of offspring that inherited and did not inherit the allele from a heterozygous parent and $P$ indicates $P$-values of the exact binomial test before correction for false discovery rate.

a Haplotype not found in GenBank. 
Finally, neither nucleotide nor amino-acid sequence divergence in offspring was significantly different from expected values obtained by Monte Carlo simulations (Table 2). We found no evidence for a non-random association between similar or dissimilar MHC alleles in our data. This was also true in families where the male and the female share one allele as an ultimate test of the complementary gene hypothesis (see Supplementary Table S3).

\section{DISCUSSION}

Our results provided no evidence for any non-random association among MHC haplotypes in gametes. We observed no significant transmission distortion of the alleles across all families or any deviation from expected allele frequencies within families. MHCsequence divergence did not deviate significantly from expected values. Although our sampling was performed at the eyed stage and hence early embryo survival was not monitored, this is unlikely to have an impact on our results. The reason for this is that if non-random gamete fusion was to occur, it would require a higher mortality rate among these offspring in order to obtain Mendelian frequencies as we found them, contradicting the idea of an adaptive scenario. Therefore, given the robustness of our data set, we can confidently exclude the existence of a mechanism ensuring complementary MHC allele combination at the haploid stage in this population of Atlantic salmon. Whether the same is true at a larger scale for example between populations remains to be tested. Our results thereby contribute to the ongoing debate on the role of MHC-based preand postmating mate choice. We discuss our results in the light of previous findings and their wider implications.

Only few studies have so far investigated the existence of mechanisms allowing for complementary MHC allele associations at the gametic haplotype level. An early study in house mice provided some experimental evidence for such a mechanism as MHC alleles exhibited non-random associations in blastocysts (Wedekind et al., 1996). These results in house mice were confirmed to be based on MHC-driven gamete association in a follow-up study (Rülicke et al., 1998) and a more recent study suggests that more general selection for heterozygosity could be driving the observed patterns (Firman and Simmons, 2015). However, whether this pattern occurs because of the complementary association of the gametes themselves or is influenced by the outcome of the second meiotic division shortly after fertilization is currently unclear. A later study in whitefish Coregonus sp. found no evidence for non-random gamete fusion in MHC-IIB (Wedekind et al., 2004). In this study, seven in vitro fertilized batches with 30-129 offspring were examined, and despite a strong effect of MHC genotype on disease resistance, the genotype frequencies in offspring were not different from expectation. However, this study did not test whether sequence divergence played any role in gamete fusion. A study on haplotype co-segregation at MHC-II in the Atlantic salmon reported that 1 out of 33 families showed segregation distortion after Bonferroni correction, suggesting that MHC was following Mendelian inheritance (Stet et al., 2002). These previous findings in fish are in line with our own findings, but drawing any general conclusions about the existence of a mechanism of MHC recognition at the gametic haplotype level would certainly be premature at this stage given the few empirical studies available. It would for example be interesting to investigate how mechanisms of gamete recognition differ between mammals and fish or other externally fertilizing taxa. For example, the interaction between sperm and ovarian fluid may be one important selection mechanism in externally fertilizing fish (Johnson et al., 2014; Rosengrave et al., 2016).
In salmonid fish, fertilization is external and gametes associate rapidly after they are released into water (Hoysak and Liley, 2001). As little as $2 \mathrm{~s}$ delay in sperm release in an in vitro competition experiment induced a significant decrease in fertilization success (Yeates et al., 2007). Yeates et al. (2009) argued that such rapid sperm-egg association makes it difficult to identify mechanisms for the recognition of the sperm MHC haplotype by the ovum. Furthermore, Wedekind et al. (2004) suggested that MHC-based non-random gamete fusion has not evolved in fish because (1) the costs of non-random gamete fusion may be higher in external fertilizers, as the gametes are released into a hostile environment with reduced time for assortative mating; (2) the selection for assortative mating is low because fish produce a much larger amount of eggs, whereas the investment per egg is lower compared with mammals; and (3) the risk of inbreeding is lower and thus there may be no selection for mechanisms to avoid inbreeding. Atlantic salmon return to their hatching grounds for spawning and hence inbreeding is likely to pose a certain risk. However, local adaptation has been shown to be under strong selection in Atlantic salmon (Dionne et al., 2008) and outbreeding might actually pose an even higher threat for the offspring survival than inbreeding (Côte et al., 2014). A topic of further investigation could therefore be to repeat the experiment using two different populations that usually do not interbreed, differing in MHC alleles, to examine whether distortion occurs with atypical fertilizations under the scope of outbreeding depression.

In conclusion, we show that MHC-II genes show no evidence of transmission ratio distortion or other signs of non-random gamete fusion in the Atlantic salmon. Our findings differ from results in studies focussing on mate choice at the premating level (reviewed in Kamiya et al., 2014; Milinski, 2014) and situations where more than two males compete for the fertilization of eggs (see, for example, Schwensow et al., 2008; Yeates et al., 2009; Lovlie et al., 2013). It appears that unlike in mammals, selection for an MHC recognition system at the haploid level may not have evolved in externally fertilizing fish. However, here we focussed on MHC-II alleles and it remains to be tested whether other MHC loci such as class I genes located on different chromosomes in fish (Sato et al., 2000) show similar inheritance patterns to those described here for class II. Furthermore, an important and currently unanswered question is whether MHC molecules are expressed at the sperm surface and whether they reflect the diploid genotype of the male or the haploid genotype of the sperm, and how these expression patterns differ across taxa.

\section{DATA ARCHIVING}

Sequence data have been submitted to GenBank: DAA: KY351821KY351823; DAB: KY351824 and KY351825.

Genotype data have been submitted to Dryad: doi:10.5061/ dryad.7kq17 The AnalyseMyData package is available at: https:// github.com/situssog/Promerova_et_al_2016.

\section{CONFLICT OF INTEREST}

The authors declare no conflict of interest.

\section{ACKNOWLEDGEMENTS}

We thank the staff of Älvkarleby salmon breeding facility for help with sampling and during egg incubation, and Holger Schielzeth for help with the statistics. Sequencing was performed at the SNP\&SEQ Technology Platform and computer resources were supplied by Uppmax. The study was financially supported by grants from the Swedish Research Council, the European Research Council and Uppsala University to SI. 


\section{AUTHOR CONTRIBUTIONS}

SI, MP and RB conceived and designed the study; SI, GA and MP did experimental work; ST extracted genotypes; and MP and RB performed statistical analyses. All authors contributed to the writing of the manuscript.

Arnaizvillena A, Festenstein H (1976). HLA genotyping by using spermatozoa - evidence for haploid gene-expression. Lancet 2: 707-709.

Agulnik SI, Sabantsev ID, Ruvinski AO (1993). Effect of sperm genotype on chromatid segregation in female mice heterozygous for aberrant chromosome 1. Genet Res 61: 97-100.

Brown JH, Jardetzky TS, Gorga JC, Stern LJ, Urban RG, Strominger JL et al. (1993) Three-dimensional structure of the human class II histocompatibility antigen HLA-DR1. Nature 364: 33-39.

Carroll LS, Penn DJ, Potts WK (2002). Discrimination of MHC-derived odors by untrained mice is consistent with divergence in peptide-binding region residues. Proc Natl Acad Sci USA 99: 2187-2192.

Consuegra S, Garcia de Leaniz C (2008). MHC-mediated mate choice increases parasite resistance in salmon. Proc $R$ Soc $B$ 275: 1397-1403.

Côte J, Roussel J-M, Le Cam S, Evanno G (2014). Outbreeding depression in Atlantic salmon revealed by hypoxic stress during embryonic development. Evol Biol 41 561-571.

Cutrera AP, Fanjul MS, Zenuto RR (2012). Females prefer good genes: MHC-associated mate choice in wild and captive tuco-tucos. Anim Behav 3: 847-857.

Dionne M, Caron F, Dodson JJ, Bernatchez L (2008). Landscape genetics and hierarchical genetic structure in Atlantic salmon: the interaction of gene flow and local adaptation. Mol Ecol 17: 2382-2396.

Dixon B, Nagelkerke L, Sibbing F, Egberts E, Stet R (1996). Evolution of MHC class II B chain-encoding genes in the Lake Tana barbel species flock (Barbus intermedius complex). Immunogenetics 44: 419-431.

Eizaguirre C, Yeates SE, Lenz TL, Kalbe M, Milinski M (2009). MHC-based mate choice combines good genes and maintenance of MHC polymorphism. Mol Ecol 18 3316-3329.

Ekblom R, Saether SA, Grahn M, Fiske P, Kalas JA, Hoglund J (2004). Major histocompatibility complex variation and mate choice in a lekking bird, the great snipe (Gallinago media). Mol Ecol 13: 3821-3828.

Frantsi C, Withey KG. (1972). A procedure for disinfecting Atlantic salmon Salmo salar eggs using a poly vinyl pyrrolidone iodine solution. Can Fish Mar Serv Res Dev Br Halifax Progr Rep 6: 1-8.

Firman RC, Simmons LW (2015). Gametic interactions promote inbreeding avoidance in house mice. Ecol Lett 18: 937-943.

Gomez D, Conejeros P, Marshall SH, Consuegra S (2010). MHC evolution in three salmonid species: a comparison between class II alpha and beta genes. Immunogenetics 62: 531-542.

Haas GG, Nahhas F (1986). Failure to identify HLA ABC and Dr antigens on human sperm. Am J Reprod Immunol Microbiol 10: 39-46.

Halim K, Festenstein H (1975). HLA-D on sperm is haploid, enabling use of sperm for HLA-D typing. Lancet 2: 1255-1256.

Hoysak DJ, Liley NR (2001). Fertilization dynamics in sockeye salmon and a comparison of sperm from alternative male phenotypes. J Fish Biol 58: 1286-1300.

Hughes AL, Nei M (1998). Pattern of nucleotide substitution at major histocompatibility complex class I loci reveals overdominant selection. Nature 335: 167-170.

Johnson SL, Villarroel M, Rosengrave P, Carne A, Kleffmann T, Lokman PM et al. (2014) Proteomic analysis of Chinook salmon (Oncorhynchus tshawytscha) ovarian fluid. PLoS One 9: e104155.

Kamiya T, O'Dwyer K, Westerdahl H, Senior A, Nakagawa S (2014). A quantitative review of MHC-based mating preference: the role of diversity and dissimilarity. $\mathrm{Mol} E \mathrm{Col} 23$ : 5151-5163.

Klein J (1986). Natural History of the Major Histocompatibility Complex. John Wiley and Sons: New York.

Kurpisz M, Fernandez N, Witt M, Kowalik I, Szymczynski GA, Festenstein H (1987). HLA expression on human germinal cells. Int J Immunogenet 14: 23-32.

Landry C, Garant D, Duchesne P, Bernatchez L (2001). 'Good genes as heterozygosity': the major histocompatibility complex and mate choice in Atlantic salmon (Salmo salar) Proc R Soc B 268: 1279-1285.
Langefors A, Lohm J, von Schantz T, Grahn M (2000). Screening of MHC variation in Atlantic salmon (Salmo salar): a comparison of restriction fragment length polymorphism (RFLP), denaturing gradient gel electrophoresis (DGGE) and sequencing. Mol Ecol 9: 215-219.

Lenz T, Wells K, Pfeiffer M, Sommer S (2009). Diverse MHC IIB allele repertoire increases parasite resistance and body condition in the long-tailed giant rat (Leopoldomys sabanus). BMC Evol Biol 9: 269.

Lohm J, Grahn M, Langefors A, Andersen O, Storset A, von Schantz T (2002). Experimental evidence for major histocompatibility complex-allele-specific resistance to a bacterial infection. Proc R Soc B 269: 2029-2033.

Lovlie H, Gillingham MAF, Worley K, Pizzari T, Richardson DS (2013). Cryptic female choice favours sperm from major histocompatibility complex-dissimilar males. Proc $R$ Soc B 280: 20131296

Magoč T, Salzberg SL (2011). FLASH: fast length adjustment of short reads to improve genome assemblies. Bioinformatics 27: 2957-2963.

Milinski M (2014). Arms races, ornaments and fragrant genes: The dilemma of mate choice in fishes. Neurosci Biobehav R 46: 567-572.

Penn DJ, Potts WK (1998). MHC-disassortative mating preferences reversed by crossfostering. Proc R Soc B 265: 1299-1306.

Piertney SB, Oliver MK (2006). The evolutionary ecology of the major histocompatibility complex. Heredity 96: 7-21.

Potts WK, Manning CJ, Wakeland EK (1991). Mating patterns in seminatural populations of mice influenced by MHC genotype. Nature 352: 619-621.

R Core Team (2014). R: A Language and Environment for Statistical Computing. R Foundation for Statistical Computing: Vienna, Austria. URL http://www.R-project.org/.

Richardson DS, Komdeur J, Burke T, von Schantz T (2005). MHC-based patterns of social and extra-pair mate choice in the Seychelles warbler. Proc $R \operatorname{Soc} B 272$ : 759-767.

Rosengrave P, Montgomerie R, Gemmell NJ (2016). Cryptic female choice enhances fertilization success and embryo survival in chinook salmon. Proc $R$ Soc $B 283$ : 20160001.

Rülicke T, Chapuisat M, Homberger FR, Macas E, Wedekind C (1998). MHC-genotype of progeny influenced by parental infection. Proc $R$ Soc Lond B 265: 711-716.

Sato A, Figueroa F, Murray BW, Málaga-Trillo E, Zaleska-Rutczynska Z, Sültman $\mathrm{H}$ et al. (2000). Nonlinkage of major histocompatibility complex class I and class II loci in bony fishes. Immunogenetics 51: 108-116.

Schwensow N, Eberle M, Sommer S (2008). Compatibility counts: MHC-associated mate choice in a wild promiscuous primate. Proc $R$ Soc $B$ 275: 555-564.

Sommer $S$ (2005). The importance of immune gene variability (MHC) in evolutionary ecology and conservation. Front Zool 2: 1-18.

Stet RJM, de Vries B, Mudde K, Hermsen T, van Heerwaarden J, Shum BP et al. (2002). Unique haplotypes of co-segregating major histocompatibility class II A and class II B alleles in Atlantic salmon (Salmo salar) give rise to diverse class II genotypes. Immunogenetics 54: 320-331.

Taborsky M (1998). Sperm competition in fish: bourgeois males and parasitic spawning. Trends Ecol Evol 13: 222-227.

Tamura K, Peterson D, Peterson N, Stecher G, Nei M, Kumar S (2011). MEGA5: molecular evolutionary genetics analysis using maximum likelihood, evolutionary distance, and maximum parsimony method. Mol Biol Evol 28: 2731-2739.

Wedekind C (1994). Mate choice and maternal selection for specific parasite resistances before, during and after fertilization. Phil Trans $R$ Soc Lond $B$ 346: 303-311.

Wedekind C, Chapuisat M, Macas E, Rülicke T (1996). Non-random fertilization in mice correlates with the MHC and something else. Heredity 77: 400-409.

Wedekind C, Seebeck T, Bettens F, Paepke AJ (1995). MHC-dependent mate preferences in humans. Proc $R$ Soc B 260: 245-249.

Wedekind C, Walker M, Portmann J, Cenni B, Muller R, Binz T (2004). MHC-linked susceptibility to a bacterial infection, but no MHC-linked cryptic female choice in whitefish. J Evol Biol 17: 11-18.

Yamazaki K, Beauchamp GK, Kupniewski D, Bard J, Thomas L, Boyse EA (1988). Familial imprinting determines H-2 selective mating preferences. Science 240: 1331-1332.

Yeates S, Searle J, Ward RG, Gage MJG (2007). A two-second delay confers first-male fertilization precedence within in vitro sperm competition experiments in Atlantic salmon. J Fish Biol 70: 318-322.

Yeates SE, Einum S, Fleming IA, Megens H-J, Stet RJM, Hindar K et al. (2009). Atlantic salmon eggs favour sperm in competition that have similar major histocompatibility alleles. Proc $R$ Soc B 276: 559-566.

Supplementary Information accompanies this paper on Heredity website (http://www.nature.com/hdy) 